\title{
UPPER SEMICONTINUOUS DECOMPOSITIONS OF THE $n$-SPHERE
}

\section{KYUNG WHAN KWUN}

We consider conditions under which an upper semicontinuous decomposition has the decomposition space which is a topological $n$ sphere. A special emphasis is placed on the case in which the decomposition has only a countable number of nondegenerate elements. If $M$ is an $n$-manifold with boundary, $\mathrm{Bd} M$ and Int $M$ will be used to denote the usual boundary and the interior of $M$. The set theoretical boundary of a subset $A$ in a space will be denoted by $b(A)$ and the closure of $A$ by $\mathrm{Cl} A$.

1. $I$-sets in $S^{n}$. A subset $F$ of the $n$-sphere $S^{n}$ is called an $I$-set if $S^{n}-F$ is homeomorphic to $S^{n}-A$ for some countable subset $A$ of $S^{n}$.

Lemмa 1. Let $A$ be a countable set in $n$-space $E^{n}, n>1$. Then $E^{n}-A$ is connected.

Lemma $1^{\prime}$. If $X$ is a connected n-manifold with boundary, $n>1$, $X-A$ is connected for any countable set $A$.

The Proof of Lemmas 1 and $1^{\prime}$. If $A$ is dense in $E^{n}$, Lemma 1 follows from Proposition A, p. 44, of [5] and the general case can be proved using this special case. For the proof of Lemma $1^{\prime}$, use Lemma 1.

Lemma 2. Each component of an I-set $F$ in $S^{n}, n>1$, is closed.

Proof. Suppose a component $C$ of $F$ is not closed. Let $x \in \mathrm{Cl} C-C$. Let $h$ be a homeomorphism of $S^{n}-F$ onto $S^{n}-A$ for some countable set $A$. Since $A$ is countable, there exist $n$-cells $D_{1}, D_{2}, \cdots$ such that Int $D_{i} \supset D_{i+1}, \operatorname{Bd} D_{i} \cdot A=\varnothing$ for each $i$ and the intersection of the $D_{i}$ is the point $h(x)$. Let $U_{i}$ be the complementary domain of $S_{i}=h^{-1}\left(\mathrm{Bd} D_{i}\right)$ containing $x$ and $U_{i}^{\prime}$ the other complementary domain. Then $\left(U_{i}-F\right)+\left(U_{i}^{\prime}-F\right)$ is a disjoint sum equal to a disjoint sum $h^{-1}\left(\right.$ Int $\left.D_{i}-A\right)+h^{-1}\left(S^{n}-A-D_{i}\right)$. Since the four sets are open in $S^{n}-S_{i}-F$ and the last two sets are connected by Lemma $1^{\prime}$, it follows that $U_{i}-F=h^{-1}\left(\right.$ Int $\left.D_{i}-A\right)$. Each $U_{i}$ contains $\mathrm{Cl} U_{i+1}$ and the intersection $U$ of the $U_{i}$ is a compact set contained in $F+x$ as the intersection of $D_{i}$ is $h(x)$. Since $C$ is connected, $U_{i}$ is open and $S_{i}$ is disjoint from $F$, each $U_{i}$ contains $C$. Since $C$ is closed in $F$, it follows that $\mathrm{Cl} C=C+x$. Let $y \in b(C)-x$. (It is impossible that $b(C)=x$.)

Received by the editors March 16, 1961. 
Let $y_{1}, y_{2}, \ldots$ be a sequence of points in $S^{n}-F$ that converges to $y$. Let $h\left(y_{i_{1}}\right), h\left(y_{i_{2}}\right), \cdots$ be a converging subsequence of $h\left(y_{i}\right)$. Then $y^{\prime}=\lim h\left(y_{i_{p}}\right)$ is on $A$. Let $E_{i}$ be $n$-cells such that Int $E_{i} \supset E_{i+1}$, $\operatorname{Bd} E_{i} \cdot A=\varnothing$ for each $i$ and the intersection of $E_{i}$ is $y^{\prime}$. Let $V_{i}$ be the complementary domain of $S_{i}^{\prime}=h^{-1}\left(\mathrm{Bd} E_{i}\right)$ containing $y$. Then $V_{i}-F=h^{-1}\left(\right.$ Int $\left.E_{i}-A\right)$ as $V_{i}$ contains infinitely many $y_{j}$. Then each $\mathrm{Cl} V_{i}$ must contain $\mathrm{Cl} C$ for the same reason as before, but this is impossible as the intersection of $E_{i}$ is disjoint from $h(x)$.

Let $F$ be any subset whatsoever of $S^{n}$. By $G(F)$ we denote the collection of the components of $F$ and the points of $S^{n}-F$.

TheOREM 1. Let $G$ be a decomposition of $S^{n}, n>1$, having only a countable number of nondegenerate elements. In order that $G$ be an upper semicontinuous decomposition of $S^{n}$ having an $n$-sphere as decomposition space, it is necessary and sufficient that $G=G(F)$ for some $I$-set $F$ in $S^{n}$.

Proof. The necessity. Let $g$ be an arbitrary element of $G$ and $X$ and $f$ respectively denote the decomposition space and the quotient map of $G$. Let $D_{1}, D_{2}, \cdots$ be $n$-cells in $X$ such that $\mathrm{Bd} D_{i}$ do not meet the image under $f$ of any nondegenerate element of $G$ and the intersection of $D_{i}$ is $f(g)$. Then $g$ is the intersection of a decreasing sequence of compact connected sets $U_{i}$, where $U_{i}$ are the closures of the complementary domains of $f^{-1}\left(\mathrm{Bd} D_{i}\right)$ containing $g$. Hence $g$ is connected. Then $G=G(F)$ for the sum $F$ of the nondegenerate elements of $G$.

The sufficiency. We first show that $G(F)$ is upper semicontinuous. Let $x \in S^{n}-F$. It is easy to see that $x$ has an arbitrarily small neighborhood which is the sum of elements of $G(F)$. (Recall the proof of Lemma 2.) Let $C$ be a component of $F$ and $h$ be a homeomorphism of $S^{n}-F$ onto $S^{n}-A$ for some countable subset $A$ of $S^{n}$. Let $x_{1}$, $x_{2}, \cdots$ be a sequence of points in $S^{n}-F$ that converges to a point $x \in C .{ }^{1}$ Then by an argument similar to one in the proof of Lemma 2, we see that $C$ is contained in the intersection of a decreasing sequence of compact connected sets $\mathrm{Cl} V_{i}$, where $V_{i}$ is a complementary domain of an $(n-1)$-sphere disjoint from $F$, and the intersection of $\mathrm{Cl} V_{\boldsymbol{i}}$ is contained in $F$. That the intersection in fact is $C$ follows from the fact that $C$ is a component of $F$ and the intersection is connected. Then a $V_{i}$ with a sufficiently large $i$ is a very close neighborhood of $C$ which is the sum of elements of $G(F)$.

We now define a map $f$ of $S^{n}$ onto itself as follows. For each point $x \in S^{n}-F$, we let $f(x)=h(x)$. For each component $C$ of $F$, let $y_{1}$, $y_{2}, \ldots$ be a sequence of points in $S^{n}-F$ that converges to a point $y \in C$. Let $h\left(y_{i_{1}}\right), h\left(y_{i_{2}}\right), \cdots$ be a subsequence of $h\left(y_{i}\right)$ that con-

1 The existence of $x$ is assured by the closedness of $C$. (See Lemma 1.) 
verges to a point $y^{\prime}$. Then $y^{\prime} \in A$ as $y_{i}$ do not have a limit point in $S^{n}-F$. Let $f(C)=y^{\prime}$. The map $f$ is then well-defined. For if $y^{\prime}$ depended on $y$, etc. and in fact had two $y^{\prime \prime}$, by an argument similar to the proof of Lemma 2, we would find $C$ contained in two disjoint neighborhoods. We can also prove that $f$ is onto and sends distinct elements of $G(F)$ into distinct points. To prove the continuity of $f$, let $D$ be an $n$-cell in $S^{n}$ such that $\operatorname{Bd} D$ is disjoint from $A$. Then $f^{-1}(\operatorname{Int} D)$ is a complementary domain of $f^{-1}(\operatorname{Bd} D)=h^{-1}(\operatorname{Bd} D)$ which is certainly open. Since each open set in $S^{n}$ is the sum of such Int $D$ 's, $f$ is continuous.

Corollary 1. An I-set $F$ in $S^{n}, n>1$, has only a countable number of components.

Corollary 2. Let $A$ and $B$ be two countable subsets of $S^{n}, n>1$. Then there exists a homeomorphism $h$ of $S^{n}$ onto itself such that $h(A)=B$ if and only if there is a homeomorphism $h^{\prime}$ of $S^{n}-A$ onto $S^{n}-B$. Moreover, $h$ can be taken as an extension of $h^{\prime}$.

Remark. Counter-examples show that Lemmas 1, 1' and 2, Theorem 1 and Corollaries 1 and 2 are false for $n=1$ and that in the statement of Corollary 2 the words "countable subsets" cannot be replaced by "arcs."

THEOREM 2. Let $G$ be an upper semicontinuous decomposition of $S^{\mathbf{3}}$ having a countable number of nondegenerate elements. If the decomposition space $X$ is a topological 3-sphere, then each element of $G$ has an open 3-cell as complement.

Proof. Let $f$ be the quotient map of $G$. We denote by $F$ the sum of the nondegenerate elements of $G$. Let $g$ be an arbitrary element of $G$. Since $f(F)$ is countable, there exist 3-cells $D_{1}, D_{2}, \cdots$ in $X$ such that Int $D_{i} \supset D_{i+1}, \operatorname{Bd} D_{i} \cdot f(F)=\varnothing$ for each $i$ and the intersection of $D_{i}$ is $f(g)$. Let $U_{i}=f^{-1}\left(\right.$ Int $\left.D_{i}\right)$. Then $U_{i}$ is the complementary domain of $S_{i}=f^{-1}\left(\mathrm{Bd} D_{i}\right)$ containing $g$. By the approximation [2] of Bing and the lemma below there exists a polyhedral 2-sphere $S_{i}^{\prime}$ separating $\mathrm{Cl} U_{i+1}$ from $S^{3}-U_{i-1}$ for each $i>1$. Hence there exist polyhedral 3-cells $E_{1}, E_{2}, \cdots$ such that Int $E_{i} \supset E_{i+1}$ for each $i$ and the intersection of $E_{i}$ is $f$. A repeated application of Theorem 1 of [6] proves the theorem.

Lemma 3. Let $S$ be an $(n-1)$-sphere in $S^{n}$ separating two connected closed subsets $A$ and $B$ in $S^{n}-S$. Then there exists a positive number $\epsilon$ such that if $h$ is an 6 -homeomorphism of $S$ onto $h(S)$ in $S^{n}-A-B$ then $h(S)$ separates $A$ from $B$. 
Proof. We first show that there is a positive number $\epsilon$ such that if $h$ is an $\epsilon$-homeomorphism mentioned in the lemma, then there is a homotopy connecting the inclusion map of $S$ into $S^{n}$ and the map $h$. Since a neighborhood of $S$ can be uniformly continuously imbedded in $E^{n}$, we may assume that $S$ is in $E^{n}$ and construct a homotopy involving a very close neighborhood of $S$. Let $D_{1}, D_{2}, \cdots, D_{k}$ be a finite number of convex $n$-cells such that $\sum$ Int $D_{i} \supset S$. Let $T$ be a triangulation of $S$ so fine that each simplex $\Delta$ of $T$ lies in an Int $D_{i(\Delta)}$. Let $\epsilon$ be a positive number such that each $\epsilon$-neighborhood of each simplex $\Delta$ of $T$ lies in Int $D_{i(\Delta)}$. Let $h$ be an $\epsilon$-homeomorphism of $S$ onto $h(S)$. Notice that $h(\Delta)$ lies in Int $D_{i(\Delta)}$. Then it is easy to construct the desired homotopy within $\sum$ Int $D_{i}$. Now we come back to $S^{n}$ and suppose that $\epsilon$ was chosen such that not only $h$ has the property mentioned at the beginning of the proof but also the homotopy takes place outside the sum of an open connected neighborhood $U$ of $A$ and an open connected neighborhood $V$ of $B$. Before proceeding any further, we refer the reader to [7, Definition 8.2 and Theorem 8.3 , p. 266]. Let $M=S^{n}-U-V$. A cycle $\Gamma$ irreducibly carried by $S$ and a cycle $\gamma$ irreducibly carried by the sum of a point of $A$ and a point of $B$ are linked. Since $\Gamma$ is homologous to $h(\Gamma)$ in $M, h(\Gamma)$ and $\gamma$ are also linked. Since $A$ and $B$ are connected and disjoint from $h(S), h(S)$ must separate $A$ from $B$.

REMARKs. A result for $S^{2}$ that is stronger than Theorem 2 is well known. While it may be true that an analogous theorem holds in $S^{n}$, we have no argument to prove it.

Suppose $F$ is a subset of $S^{3}$ having a countable number of components of which each has an open 3-cell complement. In general, $F$ is not an $I$-set. However, we can prove

THEOREM 3. Let $F$ be a subset of $S^{3}$ having only a countable number of components. Suppose $F$ is a $G$ set and $G(F)$ is upper semicontinuous. Then $F$ is an $I$-set if and only if each component of $F$ has the open 3-cell complement.

Proof. The "if" part follows from Theorem 1 and [1, Theorem 1]. The "only if" part follows from Theorems 1 and 2.

THEOREM 4. Let $F$ be the sum of a countable number of disjoint tame arcs in $S^{3}$. Then $F$ is an I-set if and only if $G(F)$ is upper semicontinuous.

Proof. Theorem 1 and [1, Theorem 3].

2. Similar $n$-cells in $S^{n}$. Let $S^{n-1}$ denote the "equator" of $S^{n}$. A topological $(n-1)$-sphere $S$ in $S^{n}$ is called tame if there exists a homeo- 
morphism of $S^{n}$ onto itself that sends $S$ onto $S^{n-1}$. A result [3] of Brown implies that $S$ is tame if and only if $S$ has a product neighborhood, i.e., the image of a homeomorphism of $S^{n-1} \times[0,1]$ into $S^{n}$ that sends $S^{n-1} \times 1 / 2$ onto $S$. If $S$ is tame, its product neighborhood can be taken in any given neighborhood of $S$. An $n$-cell $D$ in $S^{n}$ is called tame if $\mathrm{Bd} D$ is tame. Let $R_{i}, i=1,2$, be the subsets of $E^{n}$ consisting of the points $x$ with $\|x\| \leqq i$. Two $n$-cells in $S^{n}$ are called concentric if there is a homeomorphism of one onto $R_{2}$ that sends the other onto $R_{1}$. If $D_{1}$ and $D_{2}$ are concentric, we also say that $D_{1}$ is concentric to $D_{2}$ and vice versa. Two $n$-cells $D$ and $D^{\prime}$ with $D \subset D^{\prime}$ are concentric if and only if $D^{\prime}-$ Int $D$ is homeomorphic to $S^{n-1}$ $\times[0,1]$. A finite number of disjoint $n$-cells $D_{1}, \cdots, D_{k}$ in $S^{n}$ are called similar if there is an $n$-cell to which each $D_{i}$ is concentric. The following problem should be important.

The CONCENTRICITY PROBLEM. Let $D^{\prime}$ be a tame $n$-cell in $S^{n}$ containing another tame $n$-cell $D$ in its interior. Are $D$ and $D^{\prime}$ concentric?

This problem is equivalent to the following:

The SIMILARITy PROBLEM. Let $D_{1}, \cdots, D_{k}$ be disjoint tame $n$ cells in $S^{n}$. Are they similar?

These problems have the affirmative answers for $n \leqq 3$. They are open for $n \geqq 4$. We will require in certain theorems or lemmas that certain $n$-cells be similar or concentric. Such requirements may be deleted if and when either of the above problems is solved in the affirmative.

Let $D$ be an $n$-cell in $S^{n}$. An $n$-cell $D^{\prime}$ in $S^{n}$ is called an $\epsilon$-pseudoapproximation of $D$ if Int $D^{\prime}$ is an $\epsilon$-neighborhood of $D$.

Lemma 4. Let $D$ and $E$ be two tame $n$-cells in $S^{n}$ such that $D$ Int $E$. Then given a positive number $\epsilon$ there exists an $\epsilon$-pseudo-approximation $D^{\prime}$ of $D$ such that $D^{\prime}$ is concentric to $E$.

LEMma 5. Let $x_{1}, x_{2}, \cdots, x_{k}$ be distinct points in the interior of an $n$-cell $D$. Let $y_{1}, y_{2}, \cdots, y_{k}$ be distinct points in Int $D$. Then there exists a homeomorphism of $D$ onto itself such that it leaves $\mathrm{Bd} D$ pointwise fixed and sends each $x_{i}$ to $y_{i}$.

The proofs of the above two lemmas are omitted.

THEOREM 5. Let $D_{1}, \cdots, D_{k}$ be disjoint $n$-cells contained in, and concentric to, an $n$-cell $D$. Let $D_{1}^{\prime}, \cdots, D_{k}^{\prime}$ be disjoint $n$-cells contained in, and concentric to, $D$. Then there exists a homeomorphism $h$ of $D$ onto itself that moves no point of $\mathrm{Bd} D$ and takes $D_{i}$ onto $D_{i}^{\prime}$.

Proof. Let $x_{i} \in \operatorname{Int} D_{i}$ and $y_{i} \in \operatorname{Int} D_{i}^{\prime}$. By Lemma 5 , there exists a homeomorphism $h_{1}$ of $D$ onto itself that leaves each point of $\operatorname{Bd} D$ 
fixed and takes $x_{i}$ to $y_{i}$. Let $h_{2}$ be a homeomorphism of $D$ onto itself that leaves Bd $D$ pointwise fixed and pulls $h_{1}\left(D_{i}\right)$ toward $y_{i}$ such that $h_{2} h_{1}\left(D_{i}\right) \subset$ Int $D_{i}^{\prime}$. Now $h_{2} h_{1}\left(D_{i}\right)$ is concentric to $D_{i}^{\prime}$ for each $i$. Therefore there exists a homeomorphism $h_{3}$ that moves no point that is far from $\sum D_{i}^{\prime}$ and takes $h_{2} h_{1}\left(D_{i}\right)$ onto $D_{i}^{\prime}$. We finally let $h=h_{3} h_{2} h_{1}$.

Corollary. Let $D_{1}, D_{2}, \cdots, D_{k}$ be disjoint $n$-cells contained in, and concentric to, an $n$-cell $D$. Let $E_{1}, E_{2}, \cdots, E_{k}$ be disjoint $n$-cells contained in, and concentric to, an $n$-cell $E$. Then for any given homeomorphism $h$ of $\mathrm{Bd} D$ onto $\mathrm{Bd} E$, there exists an extension homeomorphism $H$ of $h$ that takes $D$ onto $E$ and each $D_{i}$ onto $E_{i}$.

THEOREM 6. Let $G$ be an upper semicontinuous decomposition of $S^{n}$, $n>1$, having a countable number of nondegenerate elements whose sum $F$ is closed. Then the decomposition space $X$ of $G$ is an $n$-sphere if and only if each element of $G$ has an open $n$-cell complement.

Proof. Suppose $X$ is an $n$-sphere. We denote the quotient map of $G$ by $f$. For given $g \in G$, there exist $n$-cells $D_{1}, D_{2}, \cdots$ in $X$ such that each $D_{i}$ is tame, Int $D_{i} \supset D_{i+1}, \operatorname{Bd} D_{i} \cdot f(F)=\varnothing$ and the intersection of $D_{i}$ is the point $f(g)$. Since each $(n-1)$-sphere $f^{-1}\left(\operatorname{Bd} D_{i}\right)$ is apart from $F$ by a positive distance, it is tame. A repeated application of Lemma 4 shows that $S^{n}-g$ is an open $n$-cell.

The converse follows, since $F$ is a $G_{\delta}$ set, from [1, Theorem 1 ]. (Actually, Bing proves his theorem for $n=3$ but the proof works for $n>3$ as well. Also, Theorem 6 can be slightly strengthened. In fact, we only need to assume that $F+A$ is closed for some countable set $A$.)

Theorem 7. Let $G$ be a monotone upper semicontinuous decomposition of $S^{n}$ and $F$ be the sum of the nondegenerate elements of $G$. Suppose for each positive number $\epsilon$, there exist a finite number of disjoint $n$-cells $D_{1}, D_{2}, \cdots, D_{k}$ such that $\mathrm{Cl} F \subset \sum$ Int $D_{i}$ and each $D_{i}$ lies in an $\epsilon$-neighborhood of an element of $G$ it contains. Then the decomposition space $X$ is a topological $n$-sphere.

Proof. Let $D_{1}, \cdots, D_{k}$ be disjoint tame $n$-cells such that $\mathrm{Cl} F$ $C \sum$ Int $D_{i}$ and each $D_{i}$ lies in a 1-neighborhood of an element of $G$ it contains. Let $e_{1}$ be a positive number smaller than $1 / 2$ such that no $2 e_{1}$-neighborhood of any $\mathrm{Bd} D_{i}$ meets $\mathrm{Cl} F$. Let $D_{1}^{\prime}, \cdots, D_{k_{1}}^{\prime}$ be disjoint $n$-cells such that $\mathrm{Cl} F \subset \sum$ Int $D_{i}^{\prime}$ and each $D_{i}^{\prime}$ lies in an $e_{1}$-neighborhood of an element of $G$ it contains. Then $\sum D_{i}^{\prime}$ $\subset \sum$ Int $D_{i}$. In general, there exist disjoint $n$-cells $D_{1}^{(p)}, \cdots, D_{\boldsymbol{k}_{p}}^{(p)}$ such that $\mathrm{Cl} F \subset \sum$ Int $D_{i}^{(p)}$, each $D_{i}^{(p)}$ lies in a $1 / 2^{p}$-neighborhood of an element of $G$ it contains and $\sum D_{i}^{(p)} \subset \sum$ Int $D_{i}^{(p-1)}$. 
If $g$ is an element of $G$ contained in $\mathrm{Cl} F$, there exists a sequence $D_{i_{0}}, D_{i_{1}}^{\prime}, \cdots, D_{i_{p}}^{(p)}, \cdots$ such that each $D_{i_{p}}^{(p)}$ contains $g$. Let $g_{p} \subset D_{i_{p}}^{(p)}$ be an element of $G$ of which $D_{i_{p}}^{(p)}$ is in a $1 / 2^{p}$-neighborhood. Let $g^{\prime} \in G$ be a limit set of $g_{p}$ 's. Then an $e$-neighborhood $U$ of $g^{\prime}$ meets infinitely many $g_{p}$. Therefore, by the upper semicontinuity of $G, U$ contains infinitely many $g_{p}$. Hence, infinitely many $D_{i_{p}}^{(p)}$ lie in $\left(e+1 / 2^{p}\right)$-neighborhoods of $g^{\prime}$. This means that the intersection of $D_{i_{p}}^{(p)}$ is $g^{\prime}$ and $g^{\prime}=g$. On the other hand, if $x \notin \mathrm{Cl} F$, there exists $p$ such that $x \notin \sum D_{i}^{(p)}$.

We now simplify the situation a little. By shrinking $D^{(p)}$ 's slightly inward, we may assume they are tame. Then using pseudo-approximations of Lemma 4 , we may assume that $D_{i}$ are similar and $D_{i}(p)$ is concentric to $D_{j}^{(p-1)}$ if the latter contains the former. Using Corollary to Theorem 5 and the method of Harrold [4], we see that $X$ is an $n$-sphere.

\section{REFERENCES}

1. R. H. Bing, Upper semicontinuous decompositions of $E^{3}$, Ann. of Math. 65 (1957), 363-374.

2. - Approximating surfaces with polyhedral ones, Ann. of Math. 65 (1957), 456-483.

3. M. Brown, $A$ proof of the generalized Schoenflies theorem, Bull. Amer. Math. Soc. 66 (1960), 74-76.

4. O. G. Harrold, Jr., A sufficient condition that a monotone image of the three-sphere be a topological three-sphere, Proc. Amer. Math. Soc. 9 (1958), 846-850.

5. W. Hurewicz and H. Wallman, Dimension theory, Princeton, 1941.

6. E. E. Moise, Affine structures in 3-manifolds. II. Positional properties of 2spheres, Ann. of Math. 55 (1952), 172-176.

7. R. L. Wilder, Topology of manifolds, Amer. Math. Soc. Colloq. Publ. Vol. 32, Amer. Math. Soc., New York, 1949.

The Seoul National University, Seoul, Korea 